\section{International Scientific Journal Theoretical \& Applied Science}

\author{
p-ISSN: 2308-4944 (print) e-ISSN: 2409-0085 (online) \\ Year: 2017 Issue: 09 Volume: 53
}

Published: $30.09 .2017 \quad \underline{\text { http://T-Science.org }}$
Ismatulla Qushayevich Khujaev

Scientific and innovation center of information and communication technologies at

Tashkent university of information technologies

Shukhratjon Komiljanovich Boltibaev

Scientific and innovation center of information and communication technologies at

Tashkent university of information technologies

SECTION 2. Applied mathematics. Mathematical modeling.

\title{
ANALYTICAL SOLUTION OF THE TASK FOR THE DAILY CHANGE OF GASODYNAMIC PARAMETERS OF THE MAIN GAS PIPELINE
}

Abstract: The paper proposes a method for applying the analytical solution for the main gas pipeline network to the task of distribution pressure and mass flow of gas in an elementary section, taking into account the daily periodicity of gas consumption for various boundary conditions with relatively to pressure and mass flow of gas and concentrated selection at internal nodes.

Key words: gas pipeline, main gas pipeline, mathematical modeling, concentrated selection, pressure, mass gas flow, computer experiment.

Language: Russian

Citation: Khujaev IQ, Boltibaev SK (2017) ANALYTICAL SOLUTION OF THE TASK FOR THE DAILY CHANGE OF GASODYNAMIC PARAMETERS OF THE MAIN GAS PIPELINE. ISJ Theoretical \& Applied Science, 09 (53): 150-154.

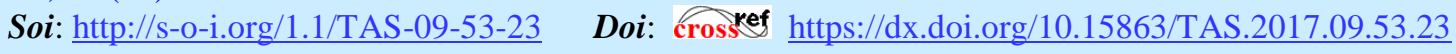

\section{АНАЛИТИЧЕСКОЕ РЕШЕНИЕ ЗАДАЧИ О СУТОЧНОМ ИЗМЕНЕНИИ ГАЗОДИНАМИЧЕСКИХ ПАРАМЕТРОВ МАГИСТРАЛЬНОГО ГАЗОПРОВОДА}

Аннотация: В работе предлагается метод применения полученные аналитического решения для сети магистрального газопроводов задачи о распространения давления и массовой расход газа на элементарном участке с учетом суточной периодичности потребления газа при различных задании граничных условий относительно давлению и массовому расходу газа и концентрированные отборы во внутренних узлах.

Ключевые слова: газопровод, магистраль газопровод, математическое моделирование, концентрированный отбор, давление, массовой расход газа, вычислительный эксперимент.

\section{Introduction}

В современных условиях развития систем магистральных газопроводов возникает необходимость разработки современных методов анализа функционирования и оперативного управления. В настоящее время существует несколько работ [1-11], посвященных проблемам расчета трубопроводных систем.

Анализ литературных источников показал $[3,4]$, что при статистической обработке обнаруживается суточную, недельную и годовую периодичность объема потребления с своими экстремумами в зависимости от состава потребителей.

Materials and Methods

Поставка газа к потребителю является постоянной, а потребление - крайне неравномерным. Неравномерность расходования газа отдельными группами потребителей определяется целым рядом факторов: сезонными климатическими изменениями, сложившимся режимом работы и отдыха трудящихся, укладом жизни населения, характеристикой газового оборудования жилых зданий и промышленных цехов. Особую остроту приобретает вопрос о покрытии пиковых расходов газа, который становится ключевым в проблеме обеспечения надежности газоснабжения. Это отмечают и зарубежные ученые. Так, P.N.Ross считает, что «уровень инвестиций в весьма капиталоемкую газовую промышленность во многом определяется величиной пикового часового потребления газа» [5]. Как утверждает И.Я.Фурман, часть пиковой потребности в газе в периоды больших похолоданий можно уменьшить за счет перевода ряда потребителей 


\begin{tabular}{|c|c|c|c|c|c|c|}
\hline Impact Factor: & $\begin{array}{l}\text { ISRA (India) } \\
\text { ISI (Dubai, UAE } \\
\text { GIF (Australia) } \\
\text { JIF }\end{array}$ & $\begin{array}{l}=1.344 \\
=0.829 \\
=0.564 \\
=1.500\end{array}$ & $\begin{array}{l}\text { SIS (USA) } \\
\text { PИНЦ (Russia) } \\
\text { ESJI (KZ) } \\
\text { SJIF (Morocco) }\end{array}$ & $\begin{array}{l}=0.912 \\
=\mathbf{0 . 2 0 7} \\
=\mathbf{3 . 8 6 0} \\
=\mathbf{2 . 0 3 1}\end{array}$ & $\begin{array}{l}\text { ICV (Poland) } \\
\text { PIF (India) } \\
\text { IBI (India) }\end{array}$ & $\begin{array}{l}=6.630 \\
=1.940 \\
=4.260\end{array}$ \\
\hline
\end{tabular}

на другие виды топлива, т.е. путем введения так называемых ограничений на газ [6].

В работах [7, 8] анализируется объем потребления природного газа в жилых зданиях с учетом температуры наружного воздуха [7] и рассматриваются вопросы определения очередей ограничения и компенсации при составлении графика лимитных ограничений для регулирования неравномерности газопотребления буферными потребителями из условия рационального распределения природного газа [8].

В работах [9-11] решены задачи о распространении периодических возмущений давления и массового расхода газа на элементарном участке газопровода и однониточной телескопической сети газопроводов с учетом концентрированных отборов и подкачек газа.

Однако эти работы, в основном, посвящены решению отдельных вопросов и не учитываются динамичность режима потребления газа по времени. Оперативное управление системами взаимосвязанных магистральных газопроводов предполагает наличие возможностей быстрого определения технологических параметров в зависимости о суточном изменении отбора газа потребителями. В связи с этим в данной работе на основе полученных ранее решений для отдельного линейного участка магистрального газопровода (дальнейшем МГ) строится решение задачи об определении параметров систем магистральных газопроводов при различных условиях их функционирования.

Рассмотрим задачу о перегоне МГ, который состоит из $N$ линейных однониточных участков. Каждый $n$-й из этих участков имеет длину $l_{n}$, внутренний диаметр $D_{n}$, коэффициент сопротивления $\lambda_{n}$, а также характерную скорость $w_{*_{n}}$, которая зависит также от газодинамических параметров участка. Кроме того, в линеаризованных параболических уравнениях для $\quad n$-го $\quad$ участка $\quad \frac{\partial p_{n, m}}{\partial t}=a_{n}^{2} \frac{\partial^{2} p_{n, m}}{\partial x^{2}} \quad$ и $\frac{\partial M_{n, m}}{\partial t}=a_{n}^{2} \frac{\partial^{2} M_{n, m}}{\partial x^{2}} \quad$ фигурируют коэффициенты $a_{n}^{2}=\frac{2 D_{n} c^{2}}{\lambda_{n} w_{*_{n}}}=\frac{c^{2}}{\lambda_{*_{n}}} \quad\left(\lambda_{*_{n}}=\frac{\lambda_{n} w_{*_{n}}}{2 D_{n}}\right)$.

На входе в МГ, что и составляет вход первого линейного участка, условия для давления и массового расхода газа составляют

$$
\begin{aligned}
& M_{0}(0, t)=v_{0,0}+\sum_{m=1}\left(\mu_{0, m} \sin \omega_{m} t+v_{0, m} \cos \omega_{m} t\right), \\
& p_{0}(0, t)=\zeta_{0,0}+\sum_{m=1}\left(\xi_{0, m} \sin \omega_{m} t+\zeta_{0, m} \cos \omega_{m} t\right) .
\end{aligned}
$$

Согласно математической модели [3] задачи в конце 1-го участка давление и массовый расход газа составляют

$$
\begin{gathered}
M_{1}\left(l_{1}, t\right)=v_{1,0}+\sum_{m=1}\left(\mu_{1, m} \sin \omega_{m} t+v_{1, m} \cos \omega_{m} t\right), \\
p_{1}\left(l_{1}, t\right)=\zeta_{1,0}+\sum_{m=1}\left(\xi_{1, m} \sin \omega_{m} t+\zeta_{1, m} \cos \omega_{m} t\right) .
\end{gathered}
$$

В этом же сечение, возможно, происходить изменение диаметра газопровода, и производится отбор/подкачка газа с интенсивностью

$$
m_{1}(t)=\beta_{1,0}+\sum_{m=1}\left(\alpha_{1, m} \sin \omega_{m} t+\beta_{1, m} \cos \omega_{m} t\right),
$$

т.е. массовый расход газа терпит конечный разрыв

$$
M_{2}(0, t)=M_{1}\left(l_{1}, t\right)+m_{1}(t) .
$$

Поэтому на входе во 2-й участок МГ условия для давления и массового расхода представляются $\quad p_{2}(0, t)=p_{1}\left(l_{1}, t\right) \quad$ и $M_{2}(0, t)=M_{1}\left(l_{1}, t\right)+m_{1}(t)$. Согласно данным условиям и решению задачи, когда заданы давление и массовый расход на входе, определяются показатели конечного сечения 2-го участка $p_{2}\left(l_{2}, t\right)$ и $M_{2}\left(l_{2}, t\right)$. В конце 2-го участка осуществляется также отбор/подкачка газа с интенсивностью

$$
m_{2}(t)=\beta_{2,0}+\sum_{m=1}\left(\alpha_{2, m} \sin \omega_{m} t+\beta_{2, m} \cos \omega_{m} t\right) .
$$

и так далее.

В начале $N$-го участка осуществляется отбор/подкачка газа с интенсивностью

$$
\begin{aligned}
m_{N-1}(t)= & \beta_{N-1,0}+ \\
& +\sum_{m=1}\left(\alpha_{N-1, m} \sin \omega_{m} t+\beta_{N-1, m} \cos \omega_{m} t\right) .
\end{aligned}
$$

Согласно этим входным данным для $N$-го участка $\quad p_{N}(0, t)=p_{N-1}\left(l_{N-1}, t\right) \quad$ и $M_{N}(0, t)=M_{N-1}\left(l_{N-1}, t\right)+m_{N-1}(t) \quad$ определяются значения давления $p_{N}\left(l_{N}, t\right)$ и массового расхода $M_{N}\left(l_{N}, t\right)$ на выходе из МГ.

Увязка входных и выходных параметров МГ легче осуществить в матричной форме, которая имеет одинаковый вид для шести различных постановках задачи. От постановки задачи зависит только процесс решения матричного уравнения. После того, как дополнены все газодинамические показатели на входе и на выходе перегона, можно восстанавливать этих показателей во всех узлах МГ.

Стационарная часть решения задачи в $n$-м участке будет представлять массовый расход $M_{C T n}(l)=M_{C T n}(0)=v_{n-1,0}$. Согласно уравнению сохранения импульса $\frac{\partial p}{\partial x}+\frac{\lambda w_{*}}{2 D F} M=0 \quad$ для значения давления в конце участка имеем

$$
p_{C T n}\left(l_{n}\right)=\zeta_{n-1,0}-\frac{\lambda_{* n} l_{n}}{F_{n}} v_{n-1,0} .
$$




\begin{tabular}{|c|c|c|c|c|c|c|}
\hline Impact Factor: & $\begin{array}{l}\text { ISRA (India) } \\
\text { ISI (Dubai, UAE } \\
\text { GIF (Australia) } \\
\text { JIF }\end{array}$ & $\begin{array}{l}=1.344 \\
=0.829 \\
=0.564 \\
=1.500\end{array}$ & $\begin{array}{l}\text { SIS (USA) } \\
\text { PИНЦ (Russia) } \\
\text { ESJI (KZ) } \\
\text { SJIF (Morocco) }\end{array}$ & $\begin{array}{l}=0.912 \\
=\mathbf{0 . 2 0 7} \\
=\mathbf{3 . 8 6 0} \\
=\mathbf{2 . 0 3 1}\end{array}$ & $\begin{array}{l}\text { ICV (Poland) } \\
\text { PIF (India) } \\
\text { IBI (India) }\end{array}$ & $\begin{array}{l}=6.630 \\
=1.940 \\
=4.260\end{array}$ \\
\hline
\end{tabular}

Периодическая часть решения рассматривается сначала для фиксированной частоты $\omega_{m}$, и далее суммированием находим решение в целом.

Заданных в начале $n$-го участка коэффициентов представим через вектор $\stackrel{\circ}{W}_{n-1, m}=\left(\begin{array}{c}\zeta_{n-1, m} \\ \xi_{n-1, m} \\ v_{n-1, m} \\ \mu_{n-1, m}\end{array}\right), \quad$ а $\quad$ на выходе участка $W_{n, m}=\left(\begin{array}{c}\zeta_{n, m} \\ \xi_{n, m} \\ v_{n, m} \\ \mu_{n, m}\end{array}\right)$.

Решение задачи массовый расход газа в конце этого участка определяется как $M_{n, m}(x, t)=\left[v_{n-1, m} \bar{R}_{K n, m}(x)-\mu_{n-1, m} \bar{I}_{K n, m}(x)-\right.$
$\left.-\frac{F_{n} \omega_{m}}{c^{2}} \xi_{n-1, m} \tilde{R}_{K n, m}(x)-\frac{F_{n} \omega_{m}}{c^{2}} \zeta_{n-1, m} \tilde{I}_{K n, m}(x)\right] \times$
$\times \cos \omega_{m} t+\left[v_{n-1, m} \bar{I}_{K n, m}(x)+\mu_{n-1, m} \bar{R}_{K n, m}(x)-\right.$
$\left.-\frac{F_{n} \omega_{m}}{c^{2}} \xi_{n-1, m} \tilde{I}_{K n, m}(x)+\frac{F_{n} \omega_{m}}{c^{2}} \zeta_{n-1, m} \tilde{R}_{K n, m}(x)\right] \sin \omega_{m} t$ а статическое давление -

$$
\begin{gathered}
p_{n, m}(x, t)=\left[\zeta_{n-1, m} \bar{R}_{K n, m}(x)-\xi_{n-1, m} \bar{I}_{K n, m}(x)-\right. \\
\left.\frac{\lambda_{* n}}{F_{n}} v_{n-1, m} \tilde{R}_{K n, m}(x)+\frac{\lambda_{* n}}{F_{n}} \mu_{n-1, m} \tilde{I}_{K n, m}(x)\right] \cos \omega_{m} t+ \\
+\left[\zeta_{n-1, m} \bar{I}_{K n, m}(x)+\xi_{n-1, m} \bar{R}_{K n, m}(x)-\frac{\lambda_{* n}}{F_{n}} \times\right. \\
\left.\times v_{n-1, m} \tilde{I}_{K n, m}(x)-\frac{\lambda_{* n}}{F_{n}} \mu_{n-1, m} \tilde{R}_{K n, m}(x)\right] \sin \omega_{m} t, \\
3 \text { Зесь } \\
\bar{R}_{K n, m}(x)=\cos \theta_{n, m} x \operatorname{ch} \theta_{n, m} x, \\
\bar{I}_{K n, m}(x)=-\sin \theta_{n, m} x \operatorname{sh} \theta_{n, m} x, \\
\tilde{R}_{K n, m}(x)=\frac{1}{2 \theta_{n, m}} \times \\
\times\left(\sin \theta_{n, m} x \operatorname{ch} \theta_{n, m} x+\cos \theta_{n, m} x \operatorname{sh} \theta_{n, m} x\right), \\
\tilde{I}_{K n, m}(x)=\frac{1}{2 \theta_{n, m}} \times \\
\times\left(-\sin \theta_{n, m} x \operatorname{ch} \theta_{n, m} x+\cos \theta_{n, m} x \operatorname{sh} \theta_{n, m} x\right) .
\end{gathered}
$$

Тогда взаимосвязь между входными и выходными показателями $n$-го участка составляет $W_{n, m}=A_{n, m} \stackrel{\circ}{W}_{n-1, m} ;$ где $\quad A_{n, m}=\left(\begin{array}{llll}a_{1,1}^{n, m} & a_{2,1}^{n, m} & a_{1,3}^{n, m} & a_{1,4}^{n, m} \\ a_{1,2}^{n, m} & a_{2,2}^{n, m} & a_{2,3}^{n, m} & a_{2,4}^{n, m} \\ a_{1,3}^{n, m} & a_{3,2}^{n, m} & a_{3,3}^{n, m} & a_{3,4}^{n, m} \\ a_{1,4}^{n, m} & a_{4,2}^{n, m} & a_{4,3}^{n, m} & a_{4,4}^{n, m}\end{array}\right)=\left\|a_{i, j}^{n, m}\right\|$,

$a_{1,1}^{n, m}=\bar{R}_{K n, m}\left(l_{n}\right)$,

$a_{1,2}^{n, m}=-\bar{I}_{K n, m}\left(l_{n}\right)$,

$a_{1,3}^{n, m}=-\frac{\lambda_{* n}}{F_{n}} \tilde{R}_{K n, m}\left(l_{n}\right)$, $a_{1,4}^{n, m}=\frac{\lambda_{* n}}{F_{n}} \tilde{I}_{K n, m}\left(l_{n}\right)$,

$a_{2,1}^{n, m}=\bar{I}_{K n, m}\left(l_{n}\right)$,

$a_{2,2}^{n, m}=\bar{R}_{K n m}\left(l_{n}\right)$,

$a_{2,3}^{n, m}=-\frac{\lambda_{* n}}{F_{n}} \tilde{I}_{K n, m}\left(l_{n}\right)$,

$a_{2,4}^{n, m}=-\frac{\lambda_{* n}}{F_{n}} \tilde{R}_{K n, m}\left(l_{n}\right)$,

$a_{3,1}^{n, m}=-\frac{F_{n} \omega_{m}}{c^{2}} \tilde{I}_{K n, m}\left(l_{n}\right)$,

$a_{3,2}^{n, m}=-\frac{F_{n} \omega_{m}}{c^{2}} \tilde{R}_{K n m}\left(l_{n}\right)$,

$a_{3,3}^{n, m}=\bar{R}_{K n, m}\left(l_{n}\right)$,

$a_{3,4}^{n, m}=-\bar{I}_{K n, m}\left(l_{n}\right)$,

$a_{4,1}^{n, m}=\frac{F_{n} \omega_{m}}{c^{2}} \tilde{R}_{K n, m}\left(l_{n}\right)$

$a_{4,2}^{n, m}=-\frac{F_{n} \omega_{m}}{c^{2}} \tilde{I}_{K n, m}\left(l_{n}\right)$,

$a_{4,3}^{n, m}=\bar{I}_{K n, m}\left(l_{n}\right), a_{4,4}^{n, m}=\bar{R}_{K n, m}\left(l_{n}\right)$.

С учетом отбора/подкачки в конце $n$-го участка в начале $n+1$-го участка МГ имеем

где $\quad \bar{\alpha}_{n m}=\left(\begin{array}{c}0 \\ 0 \\ b_{3}^{n, m} \\ b_{4}^{n, m}\end{array}\right)$

$$
\dot{\circ}_{n, m}=W_{n, m}+\bar{\alpha}_{n, m},
$$

отражает интенсивность

отбора/подкачки с синус и косинус компонентами для массового расхода; $b_{3}^{n, m}=\beta_{n, m}, \quad b_{4}^{n, m}=\alpha_{n, m}$.

Из этих отдельных решений составляются системы уравнений для МГ в целом. Для стационарной части решений имеем систему из двух неизвестных

$$
\left\{\begin{array}{l}
v_{N, 0}-v_{0,0}=\sum_{n=1}^{N-1} \beta_{n}, \\
\zeta_{N, 0}-\zeta_{0,0}+v_{0,0} \sum_{n=1}^{N} \Lambda_{n}=-\sum_{n=2}^{N} \Lambda_{n} \sum_{i=1}^{n-1} \beta_{i, 0} .
\end{array}\right.
$$

Из решения этой системы определяются стационарные части искомых решений.

Для фиксированной частоты возмущений $\omega_{m}$ следует система в матричной форме 


\begin{tabular}{|c|c|c|c|c|c|c|}
\hline Impact Factor: & $\begin{array}{l}\text { ISRA (India) } \\
\text { ISI (Dubai, UAF } \\
\text { GIF (Australia) } \\
\text { JIF }\end{array}$ & $\begin{array}{l}=1.344 \\
=0.829 \\
=0.564 \\
=1.500\end{array}$ & $\begin{array}{l}\text { SIS (USA) } \\
\text { PИНЦ (Russia) } \\
\text { ESJI (KZ) } \\
\text { SJIF (Morocco) }\end{array}$ & $\begin{array}{l}=0.912 \\
=0.207 \\
=\mathbf{3 . 8 6 0} \\
=\mathbf{2 . 0 3 1}\end{array}$ & $\begin{array}{l}\text { ICV (Poland) } \\
\text { PIF (India) } \\
\text { IBI (India) }\end{array}$ & $\begin{array}{l}=6.630 \\
=1.940 \\
=4.260\end{array}$ \\
\hline
\end{tabular}

$$
\begin{aligned}
& W_{N, m}= \\
& =A_{N, m}\left(A_{N-1, m} \ldots\left(A_{3, m}\left(A_{2, m}\left(A_{1, m} \stackrel{\circ}{W}_{0, m}\right)\right)\right) \ldots\right)+ \\
& +A_{N, m}\left(A_{N-1, m} \ldots\left(A_{3, m}\left(\begin{array}{ll}
A_{2, m} & \bar{\alpha}_{1, m}
\end{array}\right)\right) \ldots\right)+
\end{aligned}
$$

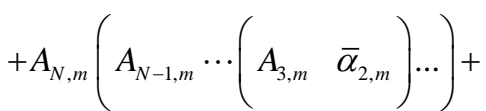

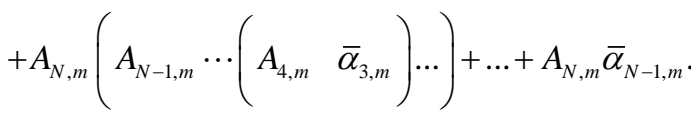

Из последнего уравнения, исключив промежуточные коэффициенты рекуррентно, получается система с четырьмя неизвестными

$$
\begin{gathered}
\left(\begin{array}{cccc}
\phi_{1}^{N, m} & 0 & 0 & 0 \\
0 & \phi_{2}^{N, m} & 0 & 0 \\
0 & 0 & \phi_{3}^{N, m} & 0 \\
0 & 0 & 0 & \phi_{4}^{N, m}
\end{array}\right)= \\
=\left(\begin{array}{c}
e_{11} \phi_{1}^{0, m}+e_{12} \phi_{2}^{0, m}+e_{13} \phi_{3}^{0, m}+e_{14} \phi_{4}^{0, m} \\
e_{21} \phi_{1}^{0, m}+e_{22} \phi_{2}^{0, m}+e_{23} \phi_{3}^{0, m}+e_{24} \phi_{4}^{0, m} \\
e_{31} \phi_{1}^{0, m}+e_{32} \phi_{2}^{0, m}+e_{33} \phi^{0, m}+e_{34} \phi^{0, m} \\
e_{41} \phi_{1}^{0, m}+e_{42} \phi_{2}^{0, m}+e_{43} \phi_{3}^{0, m}+e_{44} \phi_{4}^{0, m}
\end{array}\right)+\left(\begin{array}{l}
f_{1} \\
f_{2} \\
f_{3} \\
f_{4}
\end{array}\right) . \\
\text { Здесь }\left(\begin{array}{l}
\phi_{1}^{0, m} \\
\phi_{2}^{0, m} \\
\phi_{3}^{0, m} \\
\phi_{4}^{0, m}
\end{array}\right)=\left(\begin{array}{l}
\zeta_{0, m} \\
\xi_{0, m} \\
v_{0, m} \\
\mu_{0, m}
\end{array}\right), \quad\left(\begin{array}{l}
\phi_{1}^{N, m} \\
\phi_{2}^{N, m} \\
\phi_{3}^{N, m} \\
\phi_{4}^{N, m}
\end{array}\right)=\left(\begin{array}{l}
\zeta_{N, m} \\
\xi_{N, m} \\
v_{N, m} \\
\mu_{N, m}
\end{array}\right), \\
\tilde{f}=\bar{\alpha}_{1, m}=\left(\begin{array}{l}
0 \\
0 \\
\beta_{1, m} \\
\alpha_{1, m}
\end{array}\right),
\end{gathered}
$$

$$
\begin{aligned}
& f_{i_{N}}=\sum_{i_{N}=1}^{4} a_{i_{N}}^{N, i_{N-1}} \sum_{i_{N-1}=1}^{4} a_{i_{N-1} i_{N-2}}^{N-1, m} \times \\
& \times\left(\ldots\left(\sum_{i_{2}=1}^{4} a_{i_{3} i_{2}}^{3 m}\left(\sum_{i_{1}=1}^{4} a_{i_{2} i_{1}}^{2 m} b_{i_{1}}^{1 m}+b_{i_{2}}^{2 m}\right)+b_{i_{3}}^{3 m}\right) \ldots+b_{i_{N-1}}^{N-1, m}\right), \\
& e_{i_{N}, j}=\sum_{i_{N-1}=1}^{4} a_{i_{N}, i_{N-1}}^{N m} \sum_{i_{N-2}=1}^{4} a_{i_{N-1}, i_{N-2}}^{N-1, m} \ldots \sum_{i_{2}=1}^{4} a_{i_{3}, i_{2}}^{3 m} \sum_{i_{1}=1}^{4} a_{i_{2}, i_{1}}^{2 m} a_{i_{1}, j}^{1 m} .
\end{aligned}
$$

Из решения последней системы определяются недостающие коэффициенты давления и массового расхода в концах МГ.

\section{Conclusion}

На основе данного способа решения задач о суточном изменении газодинамических показателей МГ составлены численный алгоритм и программное средство. Составленная программа состоит из частей:

- ввода данных об участках, параметра номера задачи и соответствующих ему данных на входе и выходе МГ, данных об интенсивности отбора/подкачки в промежуточных узлах;

- формирования матриц для каждого участка и $\omega_{m}$;

- нахождения недостающих параметров в концах МГ;

- вычисления коэффициентов давления и массового расхода в промежуточных узлах МГ;

- полученные результаты вычислительных экспериментов при различных заданиях граничных условиях, позволяют принять конкретные решения по контролю функционирования системы и изменения ее параметра по времени и автоматизировать процессы гидравлических расчетов сложных систем магистральных трубопроводов.

\section{References:}

1. Sadullaev R., Khuzhaev I.K., Makhkamov M.K., Rakhimov A.Kh. (2004) Analiticheskie opredeleniya gidravlicheskikh parametrov odnonitochnogo magistral'nogo gazoprovoda pri neizotermicheskom rezhime // Metody i sistemy obrabotki informatsii: Sb. nauch. st. M.: Goryachaya liniya - Telekom, 2004. - p. 99-105.

2. Sadullaev R., Khuzhaev I.K., Boltibaev Sh.K. (2009) Izmenenie effektivnosti uchastkov gazoprovodov pri uchete sily inertsii gaza: // Sovremennye problemy mekhaniki: Materialy Mezhdunarodnoy nauchno - tekhnicheskoy konferentsii. 23-24 sentyabrya 2009. Tashkent, 2009. - p. 141-146.

3. Vodyanik P.F. (1974) Avtomatizatsiya upravleniya protsessami dobychi gaza. M.: Nedra, 1974. - 208 p.

4. Charnyy I.A. (1975) Neustanovivsheesya dvizhenie real'noy zhidkosti v trubakh. Izd. 2-e. - M.: Nedra, 1975. - 296 p.

5. Ross P.N. (1987) Designing a peak hour demand system. - Pipe Industry, 1987, vol.68, No 1, pp.55-58.

6. Furman I.Ya. (1978) Osnovnye napravleniya resheniya problemy pokrytiya pikovykh 


\begin{tabular}{l|lrl|l|ll} 
& ISRA (India) & $=\mathbf{1 . 3 4 4}$ & SIS (USA) & $=\mathbf{0 . 9 1 2}$ & ICV (Poland) & $=\mathbf{6 . 6 3 0}$ \\
Impact Factor: & ISI (Dubai, UAE) $=\mathbf{0 . 8 2 9}$ & PUHL (Russia) $=\mathbf{0 . 2 0 7}$ & PIF (India) & $=\mathbf{1 . 9 4 0}$ \\
& GIF (Australia) & $\mathbf{0 . 5 6 4}$ & ESJI (KZ) & $=3.860$ & IBI (India) & $=\mathbf{4 . 2 6 0}$ \\
& JIF & $\mathbf{1 . 5 0 0}$ & SJIF (Morocco) & $=\mathbf{2 . 0 3 1}$ & & \\
\hline
\end{tabular}

raskhodov gaza // Ekonomika gazovoy promyshlennosti. - M .: VNIIEgazprom , 1978. - № 4. - p. 3-11.

7. Tyshchik S.N., Gubar' V.F., Zakharov V.I. (2003) Analiz potrebleniya prirodnogo gaza $\mathrm{V}$ zhilykh zdaniyakh // Kommunal'noe khozyaystvo gorodov, Khar'kov, 2003, Vyp.51. - p. 149-153.

8. Tyshchik S.N. (2003) Vybor kolichestva ocheredey ogranicheniya $\mathrm{S}$ ispol'zovaniem obobshchennoy zavisimosti mezhdu urovnem gazopotrebleniya i prodolzhitel'nost'yu stoyaniya temperatur naruzhnogo vozdukha // Kommunal'noe khozyaystvo gorodov, Khar'kov, 2003, Vyp. 49. - p. 51-56.

9. Khuzhaev I.K., Boltibaev Sh.K. (2009) Periodicheskoe izmenenie gazodinamicheskikh pokazateley lineynogo uchastka gorizontal'nogo gazoprovoda pri razlichnykh granichnykh usloviyakh // Voprosy vychislitel'noy i prikladnoy matematiki. - Tashkent, 2009. Vyp. 121. - p. 100-115.

10. Sadullaev R., Khuzhaev I.K., Boltibaev Sh.K. (2010) Razrabotka obobshchennogo algoritma rascheta mnogozvennogo magistral'nogo gazoprovoda v usloviyakh sutochnoy neravnomernosti raskhoda gaza // Uzbekskiy zhurnal «Problemy informatiki i energetiki». Tashkent, 2010, №6. - p. 16-20.

11. Hujayev I.K., Boltibayev Sh.K. The algorithm for calculation of multilink gas pipeline which is functioning in periodic mode // European Applied Sciences, September, 2013, 9 (1). - p. 110-115. 